\title{
Applied research of a four-dimensional CT localization technique in radiotherapy and treatment planning for early lung cancer
}

\author{
Gang Wang", Xiwen Zhu", Fan Zhang, Jing Liu, Lu Yue, Lihua Deng, Mi Zhou \\ Department of Radiotherapy, Qingdao Municipal Hospital, Qingdao, China \\ Contributions: (I) Conception and design: M Zhou; (II) Administrative support: X Zhu, L Deng; (III) Provision of study materials or patients: G \\ Wang, L Yue; (IV) Collection and assembly of data: F Zhang; (V) Data analysis and interpretation: J Liu; (VI) Manuscript writing: All authors; (VII) \\ Final approval of manuscript: All authors. \\ \#These authors contributed equally to this work. \\ Correspondence to: Mi Zhou. Department of Radiotherapy, Qingdao Municipal Hospital, Qingdao, China. Email: 25206203@qq.com.
}

\begin{abstract}
Background: To study the feasibility of target contouring and the potential benefits to radiotherapy of four-dimensional computed tomography (4D-CT) for early stage lung cancer.

Methods: We applied Brilliance CT to scan 24 lung cancer patients for 4D localization. Treatment plannings based on different breath phase CT images were designed in the Monaco 5.2 treatment planning system. Different planning was compared to find the difference of target volume, center, and dose.

Results: Target volume composed of 10-breath phases (Sum) was considered as the reference volume, which was slightly larger than the volume contouring obtained by the maximum intensity projection (Mip) and extreme phase images (Exs). Target centers for Sum, Mip and Exs of CT images showed little deviation in the X, Y and Z directions. The V20 and V5 for the 4D-CT-based treatment plan showed a markedly lower dose delivered to normal tissues than those based on 3D-CT.
\end{abstract}

Conclusions: For target contouring, a target contouring by 10-breath phases was superior to Mip and Exs. As the gross target volume (GTV) contouring obtained by the maximum intensity projection and extreme phase were similar to that of the reference target, they can support the contouring target and reduce the time and work. Treatment plan comparisons indicated that normal lung tissues received a remarkably lower dose when a 4D-CT-based plan was used.

Keywords: Early stage lung cancer; four-dimensional computed tomography (4D-CT); target contouring; dose advantages

Submitted Jul 27, 2020. Accepted for publication Oct 27, 2020.

doi: $10.21037 /$ tcr-20-2800

View this article at: http://dx.doi.org/10.21037/tcr-20-2800

\section{Introduction}

Lung cancer has the highest morbidity among all cancers and is a grievous threat to human health and well-being (1). The 2017 China Cancer Register Annual Report reported cancer morbidity to be almost $2.95 \%$, with lung cancer morbidity and death rate being $18.93 \%$ and $25.24 \%$, respectively. For the past few decades, radiotherapy has been the primary treatment technology for lung cancer (2,3). Recently, with the development of linear accelerators and networks, image-guided radiation therapy (IGRT) and stereotactic body radiotherapy (SBRT) (4) have found broader and deeper application in the treatment of lung cancer, although significant challenges and limitations to these modalities remain. One of the challenges that has become increasingly relevant in radiation therapy for early stage lung cancer is tumor motion $(5,6)$. The radiation dose delivered to the target can be easily affected by shifts in tumor location caused by the movements of breathing. Among the techniques that control for lung cancer tumor motion, the four-dimensional computed tomography (4D-CT) localization technique has advantages in dose coverage and complication reduction (7-10). 
The goal of radiotherapy is to deliver a deadly dose of radiation to the cancer cells while leaving normal tissue relatively unharmed. In lung cancer however, this balance is hard to achieve. Thus, precisely determining the tumor volume and location is required to achieve adequate dose coverage and decrease the rate of radiation pneumonia (11-13). Despite our improved understanding of the respiratory motion and technical advancement in the management of respiratory motion during the course of radiotherapy in the past decade, many radiotherapy centers in the world continue to use free-breathing CT (FBCT) imaging for radiotherapy planning in the treatment of lung cancer. 4D-CT scan can encompass the whole tumor trajectory during a breathing cycle while maintaining high-quality image resolution. The 4D-CT scan not only precisely determines the average tumor motion but more importantly depicts the range of tumor motion in relation to surrounding normal structures. Modern 4D-CT scanners can image the whole thorax while capturing all the phases of respiratory cycle in less than a minute (14-18). We therefore aimed to investigate three types of tumor volumes and target centers in CT images, Mip, Exs, and Sum, which consisted of a compilation of 10 breath phase images captured by 4D-CT. Furthermore, a traditional 3D-CTbased treatment plan was compared to one based in the 4DCT Sum phase, in order to evaluate volume differences and the dose volume histogram. We present the following article in accordance with the MDAR reporting checklist (available at http://dx.doi.org/10.21037/tcr-20-2800).

\section{Methods}

\section{Patients materials}

Between June 2017 and October 2017, our study enrolled 24 early stage lung cancer patients, including 13 male patients and 11 female patients aged 49 to 73 years old. The patients had no lymphatic metastasis and distant metastasis, and were deemed eligible for the study by the local hospital ethics committee. Patient characteristics are displayed in Table 1. The study was conducted in accordance with the Declaration of Helsinki (as revised in 2013). The study was approved by ethics committee of Qingdao Municipal Hospital (No. 2020067). The Institutional Review Board (IRB) of our hospital waived the need for obtaining informed consent from the patients.

\section{Tumor localization}

Tumor localization was processed by a Philips Brilliance
CT scanner using an $85-\mathrm{cm}$ aperture and a $60-\mathrm{cm}$ field of view (FOV). Bellows generated breath signals according to the vital capacity and provide breath frequency, phase, and range. The DICOM images carrying the breath information were then imported into the Monaco 5.2 treatment planning system developed by Elekta (Stockholm, Sweden). This system adopts the Monte Carlo algorithm, which is known as the most accurate dose calculation method. Configuring the initial prescription in the optimization process is crucial for creating a treatment plan. Once the optimization has begun, the medical physicist cannot intervene, unless some parameters do not meet the radiation oncologists specification.

The 24 patients were scanned both in 3D and 4D CT with the same scan range: from the cricothyroid membrane to the costophrenic angle of the inferior margin. The slice thickness was $3 \mathrm{~mm}$. After tumor location scanning was completed, the in-built Brilliance CT software collected the scan information and reconstructed 10-phase CT images for the 4D-CT scan, which were named the following: $\mathrm{CT}_{0}$, $\mathrm{CT}_{10}, \mathrm{CT}_{20}, \mathrm{CT}_{30}, \mathrm{CT}_{40}, \mathrm{CT}_{50}, \mathrm{CT}_{60}, \mathrm{CT}_{70}, \mathrm{CT}_{80}$, and $\mathrm{CT}_{90}$. The CT maximal intensity projection $\left(\mathrm{CT}_{\text {Mip }}\right)$, minimum intensity projection $\left(\mathrm{CT}_{\mathrm{MinIP}}\right)$, average intensity projection $\left(\mathrm{CT}_{\mathrm{AvgIP}}\right)$, and the $\mathrm{CT}$ compilation of $\mathrm{CT}_{0}$ and $\mathrm{CT}_{50}\left(\mathrm{CT}_{\mathrm{Exs}}\right)$ were generated according to the 10 phase images. However, if the density of the tumor was greater than that of the lung, the MinIP and AvgIP were considered meaningless. Finally, 3D-CT, 10-phase, Mip, and Exs images were transferred into the Monaco 5.2 treatment planning system. Figure 1 shows one patient's 10 -phase lung images at a fixed slice.

\section{Tumor contouring}

The radiation oncologist contoured the tumor in the $3 \mathrm{D}$ and 4D images imported from the CT software. The lung window's width and level was set to 1,600 and $600 \mathrm{HU}$, respectively. According to report 62 from the International Commission on Radiation Units and Measurements (ICRU) (19), the following are the target (primary lesion excluding lymph gland) contours in different CT images: tumor contour in $3 \mathrm{D}$ images is defined as gross tumor volume $(\mathrm{GTV})_{\mathrm{C} 1}$; targets drawn in every phase are $\mathrm{GTV}_{0}$, $\mathrm{GTV}_{10}, \mathrm{GTV}_{20} \ldots \mathrm{GTV}_{80}, \mathrm{GTV}_{90}$; targets outlined in $\mathrm{CT}_{\text {Mip }}$ and $\mathrm{CT}_{\mathrm{Exs}}$ are named $\mathrm{GTV}_{\text {Mip }}$ and $\mathrm{GTV}_{\mathrm{Exs}}$; target volumes following the same naming rules are $\mathrm{V}_{\mathrm{Cl}}, \mathrm{V}_{\text {Sum }}$ (compilation of 10 GTV phases), $V_{\text {Mip }}$, and $V_{\text {Exs }}$. Analysis was performed to determine the best method to contour targets.

The center of the tumor generated by the treatment 
Table 1 Information of 24 lung cancer patients

\begin{tabular}{|c|c|c|c|c|c|c|}
\hline Patient No. & Gender & Age (years) & Tumor location & $\mathrm{T}$ & $\mathrm{N}$ & M \\
\hline 2 & M & 52 & Lung $\mathrm{R}$ inferior lobe & $1 a$ & 0 & 0 \\
\hline 3 & $\mathrm{~F}$ & 73 & Lung $L$ inferior lobe & $1 b$ & 0 & 0 \\
\hline 4 & M & 72 & Lung $R$ middle lobe & $1 b$ & 0 & 0 \\
\hline 6 & M & 52 & Lung $R$ middle lobe & $1 a$ & 0 & 0 \\
\hline 7 & M & 49 & Lung L inferior lobe & $1 a$ & 0 & 0 \\
\hline 8 & $\mathrm{~F}$ & 53 & Lung L inferior lobe & $1 b$ & 0 & 0 \\
\hline 9 & M & 53 & Lung $\mathrm{R}$ inferior lobe & $1 b$ & 0 & 0 \\
\hline 12 & M & 50 & Lung L superior lobe & $1 a$ & 0 & 0 \\
\hline 13 & M & 60 & Lung $L$ inferior lobe & $1 a$ & 0 & 0 \\
\hline 14 & $\mathrm{~F}$ & 51 & Lung $R$ middle lobe & $1 a$ & 0 & 0 \\
\hline 15 & $\mathrm{~F}$ & 49 & Lung $\mathrm{R}$ inferior lobe & $1 b$ & 0 & 0 \\
\hline 16 & M & 66 & Lung L superior lobe & $1 a$ & 0 & 0 \\
\hline 17 & M & 73 & Lung $R$ middle lobe & $1 a$ & 0 & 0 \\
\hline 18 & $\mathrm{~F}$ & 59 & Lung $\mathrm{R}$ inferior lobe & $1 b$ & 0 & 0 \\
\hline 19 & $\mathrm{~F}$ & 56 & Lung L superior lobe & $1 a$ & 0 & 0 \\
\hline 24 & $\mathrm{~F}$ & 64 & Lung $\mathrm{R}$ inferior lobe & $1 b$ & 0 & 0 \\
\hline
\end{tabular}

F, female; M, male; R, right; L, left.

planning systems (TPS) can roughly describe its location and motion. Target centers in $\mathrm{CT}_{\text {Mip }}$ and $\mathrm{CT}_{\mathrm{Exs}}$ were compared to $\mathrm{CT}_{\text {Sum }}$ to analyze position difference. While early stage lung cancer patients usually have small target volumes, the organs at risk, including normal lung tissues, can be well protected. However, considering the variable sensitivity of lung tissue to radiation and the possibility of cancer recurrence, some patients require secondary radiotherapy. We thus formulated two plan types, one based on 3D-CT and the other on 4DCT, to compare target volumes and evaluate the V20 and V5 differences of the affected lung tissue (lung volumes that accept more than 20 and 5 Gy dose, respectively).

\section{Statistical analysis}

SPSS 19.0 was used to analyze the data. When the $\mathrm{P}$ value $<0.05$, the difference was considered to be statistically significant.

\section{Results}

\section{Target volumes comparison}

Table 2 shows the ratio of different target volumes to $\mathrm{V}_{\text {Sum }}$. For the 24 patients, the $\mathrm{V}_{\mathrm{Cl}} / \mathrm{V}_{\text {Sum }}$ was approximately equal to 0.5 . The target volumes for different phases had the same 


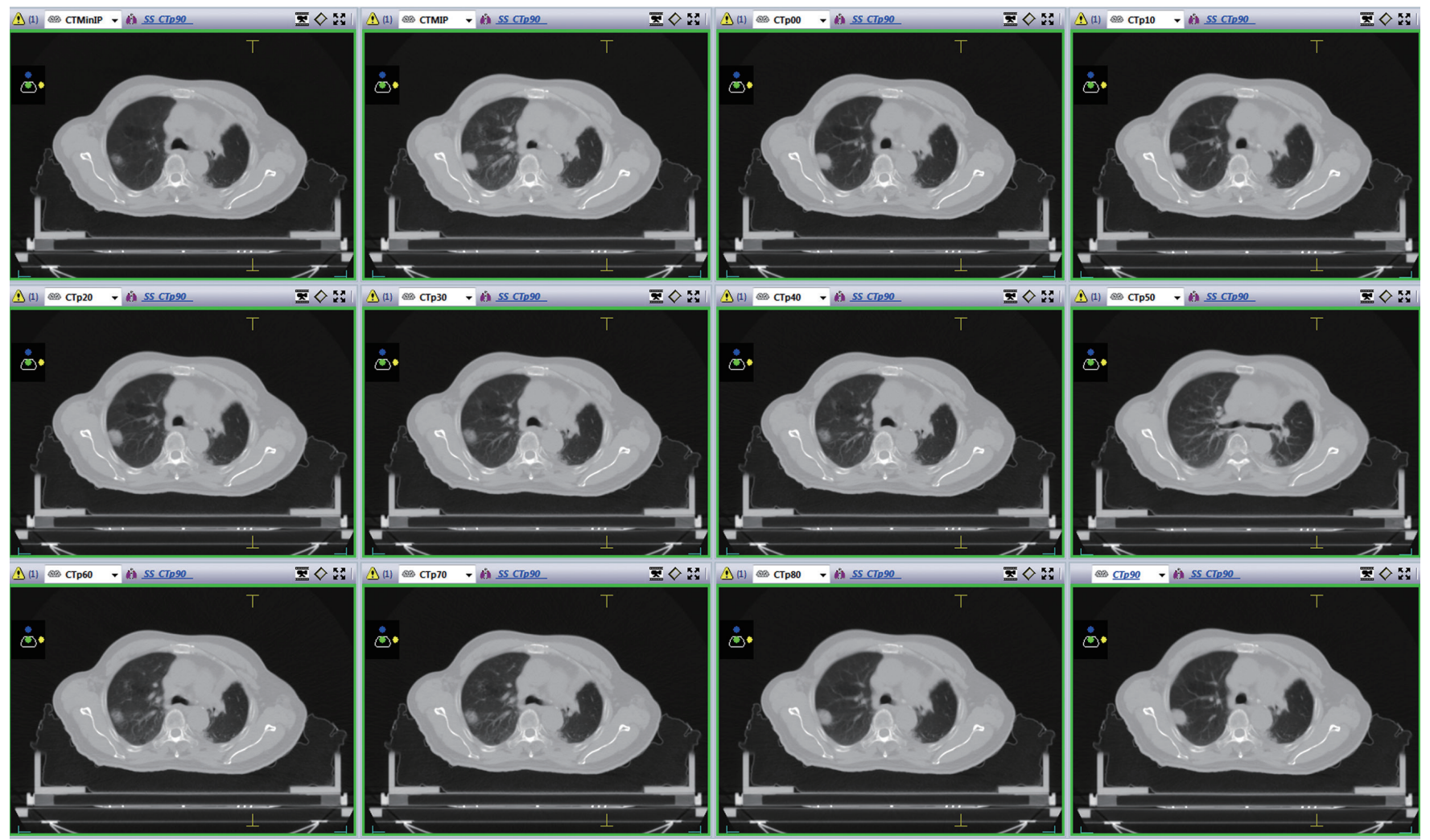

Figure 1 Ten-phase lung images from one patient.

0.5 ratio values. $V_{\text {Mip }} / \mathrm{V}_{\text {Sum }}$ and $\mathrm{V}_{\mathrm{Exs}} / \mathrm{V}_{\text {Sum }}$ were $0.888 \pm 0.061$ and $0.883 \pm 0.064$, respectively, while $V_{\text {Mip }}$ and $V_{\text {Exs }}$ were similar in value to the $V_{\text {Sum }}$.

\section{Deviations of the target center compared to $C T_{\text {Sum }}$}

The planning system automatically calculated the target center when importing the CT information. Target centers for $\mathrm{CT}_{\mathrm{Mip}}$ and $\mathrm{CT}_{\mathrm{Exs}}$ were chosen to analyze the deviations on the basis of $\mathrm{CT}_{\text {Sum. }}$. The results are shown in Table 3.

\section{Comparison of $V_{c t v}$ and $V_{\text {Sum }}$}

As we wanted to evaluate the potential dose advantage of 3D-CT-based versus 4D-CT-based plans, $\mathrm{V}_{\text {ctv }}$ was created with a $0.5 \mathrm{~cm}$ margin from the $\mathrm{V}_{\mathrm{C} 1}$. The results in Figure 2 show that $V_{\text {ctv }}$ has a larger volume than $V_{\text {Sum }}$, but the difference varies depending on each patient, which was not the case in the comparison between $V_{C 1}$ and $V_{\text {Sum. }}$. For the plan based on $V_{\text {ctv }}$ and $V_{\text {Sum }}$, targets and organs at risk (OARs) endured different doses. The details are shown in next section.

\section{V20 and V5 for Plan ctv $_{\text {and }}$ Plan}

Planning target volumes (PTVs) on Plan $_{\text {ctv }}$ and Plan $_{\text {Sum }}$ were obtained by creating a $0.5 \mathrm{~cm}$ margin from $V_{\text {ctv }}$ and $V_{\text {Sum }}$. Targets had both a $100 \%$ dose coverage and 95\% volume, while the OARs were within standard limits. Our attention was mainly focused on the dose delivered to normal lung tissue, as this not only has a significate effect on normal lung function but can indicate the radiation pneumonia rate. Figure 3 shows V5 and V20 for $\mathrm{Plan}_{\mathrm{ctv}}$ and $\mathrm{Plan}_{\text {Sum. }}$. Both V5 and V20 are lower in $\mathrm{Plan}_{\text {Sum. For Plan }}$ ctv the mean V5 and V20 is $42.798 \%$ and $21.161 \%$, respectively, while that for $\mathrm{Plan}_{\text {Sum }}$ is $31.610 \%$ and $14.950 \%$, respectively. The results indicated that the 4D-CT-based plan had an advantage in dosage, with increased tumor tissue dose coverage and a reduction in the complication rate, especially for radiation pneumonia.

\section{Discussion}

Early stage lung cancer patients and relatives can usually 
Table 2 The ratio of different target volumes to $V_{\text {Sum }}$

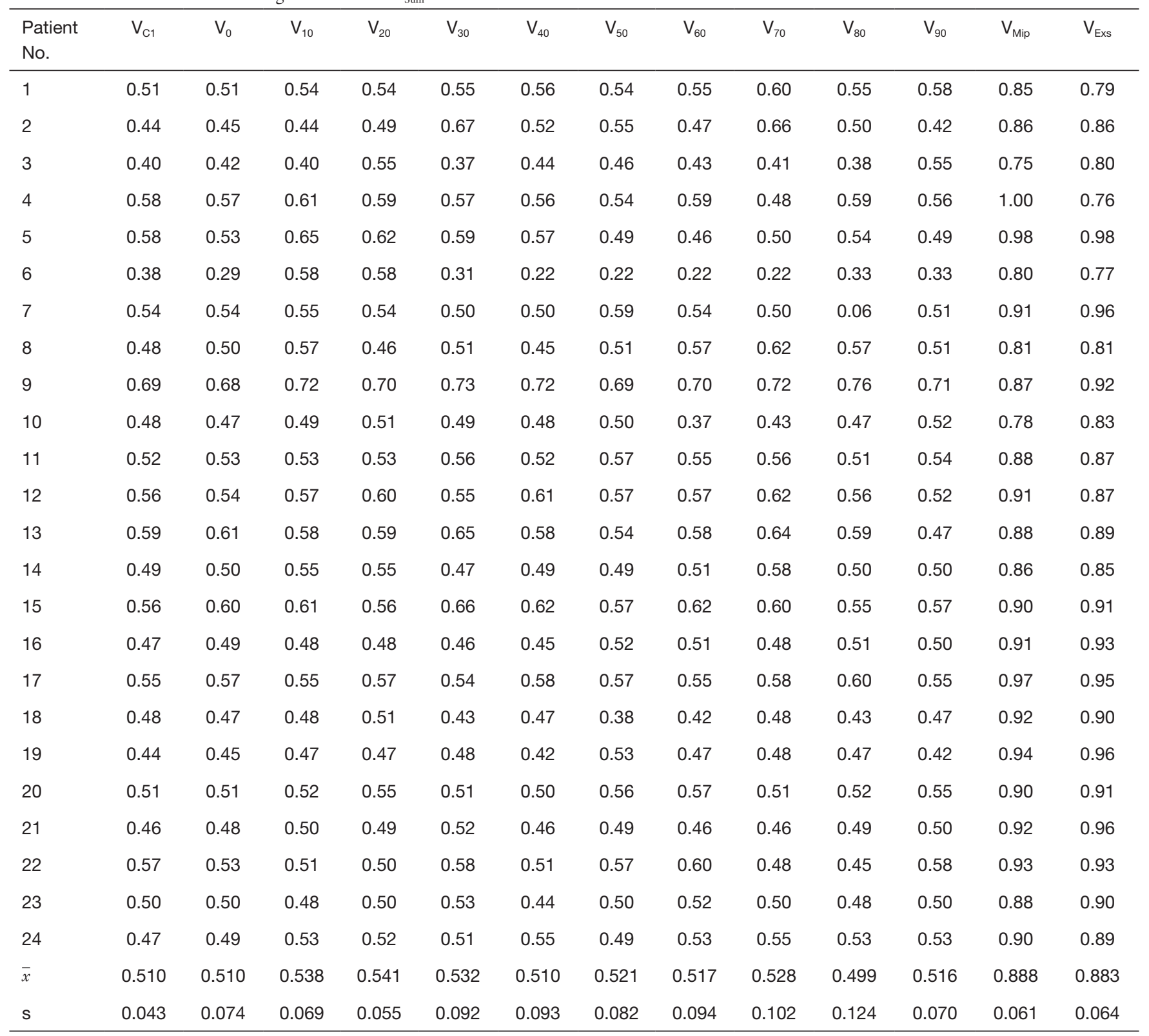

$\bar{x}$ is the mean value, $\mathrm{s}$ is the standard deviation.

expect a long period of overall survival. This requires that the whole tumor responds to a radical radiation dose, but the reality of treatment is that this cannot be guaranteed. As the tumor is usually small and isolated, it can be easily shifted by the movements of breathing. When a traditional CT location technique is adopted, the images can reflect the tumor shape and size only in a certain static state but not throughout the whole breath cycle. As a result of this motion effect, the tumor may not receive a sufficient radiation dose which may allow for the tumor to metastasize and recur. Another disadvantage of $3 \mathrm{D}$ location is that the OAR dose is increased, especially in lung tissues. As we know, the radiation oncologist's greatest concern lies in improving the target dose coverage. To achieve this goal, the oncologist must generally contour more GTV volume and expand the coverage area.

Meanwhile, as 4D-CT techniques include timing information, they can more precisely reflect the motion 


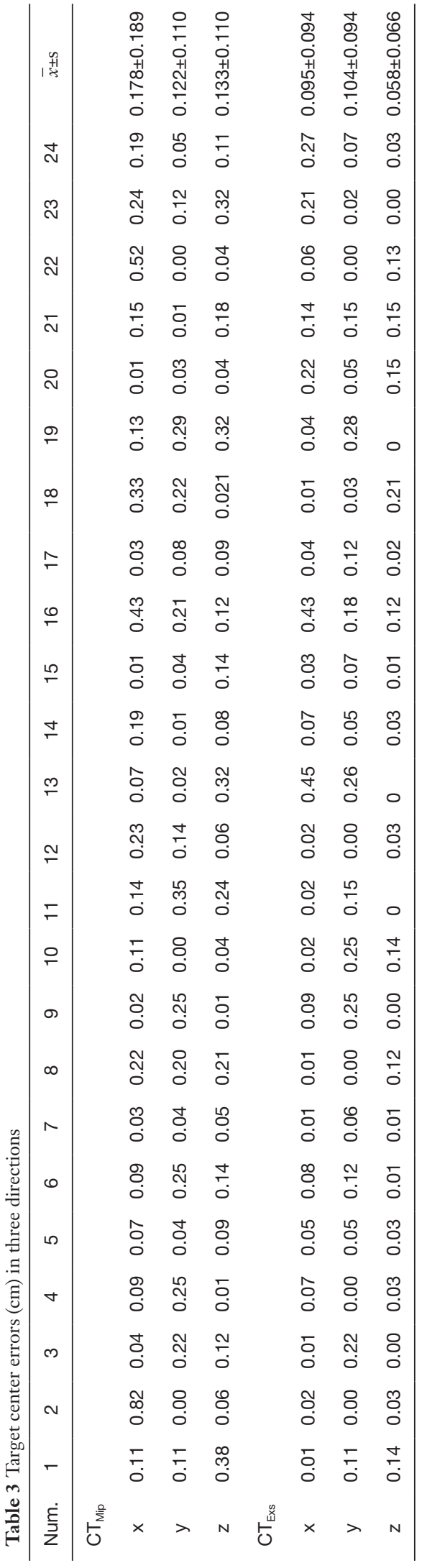

of thoracic and abdominal tumors. This approach is able to record the tumor motion trajectory and acquire the subsequent dynamic CT series. It can also track the target and OAR location and shape throughout the entire breath cycle. This ability can increase the target dosage while decreasing that of the normal tissue. Therefore, generally speaking, the emergence of $4 \mathrm{D}-\mathrm{CT}$ has resolved the issues related to the collection of information during free breathing.

In our study, the average target volume of each phase for the 24 patients was almost half of the $V_{\text {Sum }}$, which was approximately equal to the average $V_{C 1}$. The $V_{\text {Mip }}$ and $V_{\text {Exs }}$ were nearly equal to $V_{\text {Sum }}$. Thus, it appears that not every phase of CT is suitable for target contouring of the breath effect. When using $\mathrm{CT}_{\text {Mip }}$ images for contouring, Muirhead et al. (20) found that this approach was more reliable for I stage non-small cell cancer, while Huang et al. (21) reported that $\mathrm{CT}_{\text {Mip }}$ can improve the target coverage especially for large and irregular target motion. Although a target volume based on $\mathrm{CT}_{\text {Sum }}$ images has the most accuracy, it is also timeconsuming; in contrast, $\mathrm{CT}_{\text {Mip }}$ and $\mathrm{CT}_{\mathrm{Exs}}$ images can be adopted to contour the target and keep the accuracy both in volume and dose. For target volumes derived from 3D-CT and 4D-CT, Franks et al. (22) investigated the target coverage for SBRT in these two types CT images, and observed significant decreases in target coverage (V100, 0.84 vs. 0.94). In our work, the comparison of $V_{c t r}$ and $V_{\text {Sum }}$ showed that $\mathrm{V}_{\mathrm{ctv}}$ was larger than $\mathrm{V}_{\text {Sum }}$ in every patient, meaning $\mathrm{V}_{\mathrm{ctv}}$ had a bigger PTV with more dose delivered to the lungs.

The target center in the two reconstruction images, $\mathrm{CT}_{\text {Mip }}$ and $\mathrm{CT}_{\mathrm{Exs}}$, showed little difference compared to that in $\mathrm{CT}_{\text {Sum }}$. Breathing induces tumor motion, which makes it difficult to discern the exact border of the target, but has a negligible impact on its location. Respiratory gating based on 4D-CT has become increasingly important in lung cancer treatment. Research suggests that 4D-CT has advantages over 3D-CT in evaluating the target location. The 4DCT technique can ascertain the true status of a tumor affected by breathing motion and reflect the tumor motion range by eliminating the motion artifact. In our study, the target center errors in $\mathrm{x}, \mathrm{y}, \mathrm{z}$ directions were identified by comparing $\mathrm{CT}_{\text {Mip }}$ to $\mathrm{CT}_{\text {Sum }}$ which yielded the following: $0.178 \pm 0.189,0.122 \pm 0.110,0.133 \pm 0.110$; meanwhile, the values obtained from comparing $\mathrm{CT}_{\mathrm{Exs}}$ to $\mathrm{CT}_{\text {Sum }}$ were the following: $0.095 \pm 0.094,0.104 \pm 0.094$, and $0.058 \pm 0.066$. The centers were close to each other in all three image types.

The final goal for lung cancer radiation treatment is delivering a high dose of radiation to the target while 


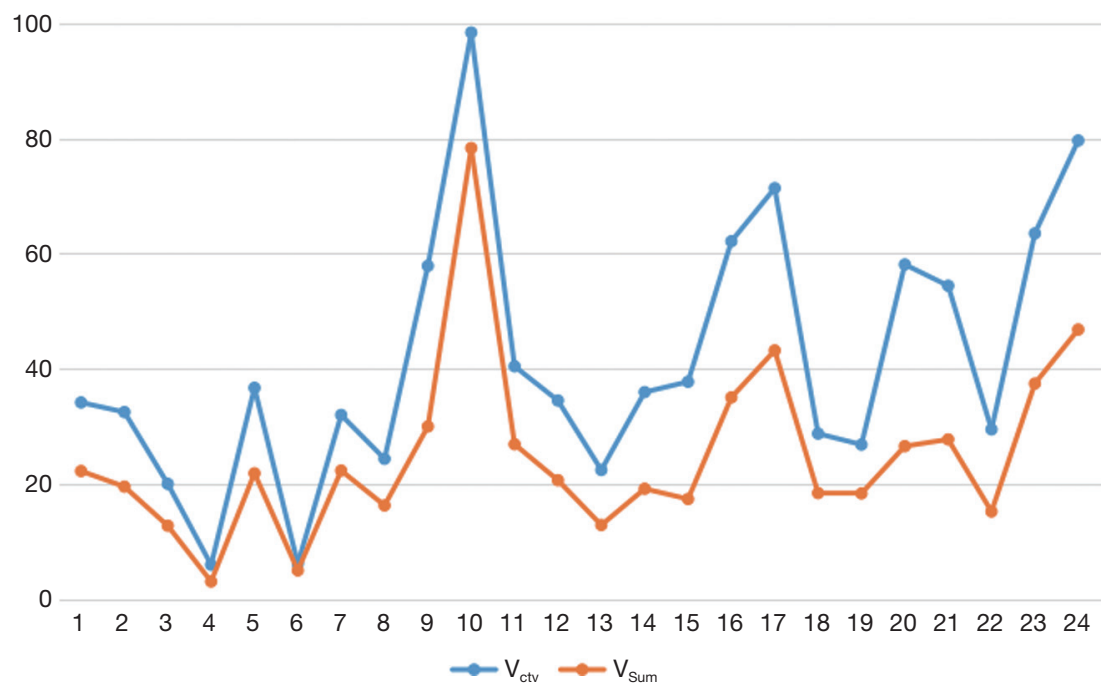

Figure 2 Comparison of $\mathrm{V}_{\mathrm{ctv}}$ and $\mathrm{V}_{\mathrm{Sum}}$.

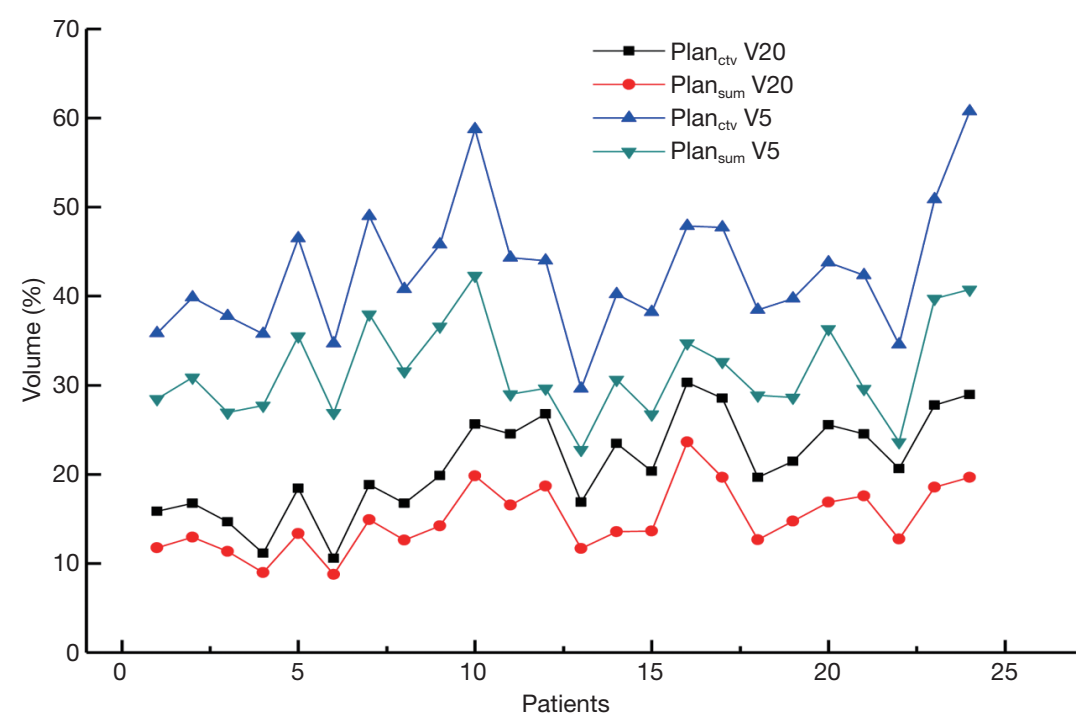

Figure 3 V20 and V5 for $\mathrm{Plan}_{\mathrm{ctv}}$ and $\mathrm{Plan}_{\mathrm{Sum}}$.

minimizing the dose delivered to OARs. For radiation treatment of thoracic tumors, the lung tissue inevitably suffers an incidental dosage $(23,24)$, and radiation damage to the lung commonly occurs because this tissue is very sensitive to the X-rays. V20 and V5 mentioned above are very important indexes for estimating lung damage risk $(13,25,26)$. In our work, two plans based on two PTV types (derived from 3D-CTV and 4D-GTV respectively) were used. With a smaller PTV volume, Plan Sum $_{\text {has an advantage }}$ in protecting lungs and decreasing the rate of radiation pneumonia.
The sample we adopted was 24 patients and closed to the same kind research. On account of 13 CT images contouring for one patient, the 24 patients were total 312 fractions. In the next work, we will add more patients to verify our results. The potential point of our work is that we didn't involve the pathological comparison for dose, target volume and target center. We find that the combination of 4D CT technology and respiratory gating can make the dose more accurate and decrease the normal lung tissue dose than one single technology. And that is our department future direction. 


\section{Conclusions}

Our study contoured the target volumes in $3 \mathrm{D}-\mathrm{CT}$ and 4D-CT $\left(\mathrm{CT}_{\mathrm{Mip}}, \mathrm{CT}_{\mathrm{Exs}}\right.$ and $\left.\mathrm{CT}_{\text {Sum }}\right)$ images for early stage lung cancer patients to identify any volume differences and target center deviations. Treatment plans based on 3D-CT and $4 \mathrm{D}-\mathrm{CT}_{\text {Sum }}$ were compared to quantitatively measure the lung tissue affected by the difference in dosage. For GTV contouring, the 10-phase $\mathrm{CT}_{\text {Sum }}$ was found to be the optimal image for contouring, while volumes contoured in $\mathrm{CT}_{\mathrm{Mip}}$ and. $\mathrm{CT}_{\mathrm{Exs}}$ showed little difference. Considering the workload in contouring in 10-phase CT images, these two types of images can also be used to contour the target. Target centers calculated for the $\mathrm{CT}_{\text {Mip }}, \mathrm{CT}_{\mathrm{Exs}}$, and $\mathrm{CT}_{\text {Sum }}$ in TPS were very similar. V5 and V20 for $4 \mathrm{D}-\mathrm{CT}_{\text {Sum }}$ were both lower than those for 3D-CT, and this can have a significant effect on decreasing radiation pneumonia and protecting the OARs.

\section{Acknowledgments}

Funding: None.

\section{Footnote}

Reporting Checklist: The authors have completed the MDAR reporting checklist. Available at http://dx.doi.org/10.21037/ tcr-20-2800

Data Sharing Statement: Available at http://dx.doi. org/10.21037/tcr-20-2800

Conflicts of Interest: All authors have completed the ICMJE uniform disclosure form (available at http://dx.doi. org/10.21037/tcr-20-2800). The authors have no conflicts of interest to declare.

Ethical Statement: The authors are accountable for all aspects of the work in ensuring that questions related to the accuracy or integrity of any part of the work are appropriately investigated and resolved. The study was conducted in accordance with the Declaration of Helsinki (as revised in 2013). The study was approved by ethics committee of Qingdao Municipal Hospital (No. 2020067). The Institutional Review Board (IRB) of our hospital waived the need for obtaining informed consent from the patients.
Open Access Statement: This is an Open Access article distributed in accordance with the Creative Commons Attribution-NonCommercial-NoDerivs 4.0 International License (CC BY-NC-ND 4.0), which permits the noncommercial replication and distribution of the article with the strict proviso that no changes or edits are made and the original work is properly cited (including links to both the formal publication through the relevant DOI and the license). See: https://creativecommons.org/licenses/by-nc-nd/4.0/.

\section{References}

1. Torre LA, Siegel RL, Jemal A. Lung cancer statistics. Adv Exp Med Biol 2016;893:1-19.

2. Kong FM, Ten Haken RK, Scliipper MJ, et al. High-dose radiation improved local tumor control and overall survival in patients with inoperable/unresectable non-small-cell lung cancer: long-term results of a radiation dose escalation study. Int J Radiat Oncol Biol Phys 2005;63:324-33.

3. Ren J, Gong G, Yao X, et al. Dosimetric comparison of dose accumulation between rigid registration and deformation registration in intensity-modulated radiation therapy for large volume non-small cell lung cancer. Trans Cancer Res 2019;8:2878-85.

4. MacManus M, De Abreu Lourenco R, Hegi-Johnson F. Helping patients with lung cancer choose between surgery or stereotactic body radiotherapy: the importance of the patient experience. J Thorac Dis 2019;11:4404-7.

5. Dhont J, Vandemeulebroucke J, Burghelea M, et al. The long- and short-term variability of breathing induced tumor motion in lung and liver over the course of a radiotherapy treatment. Radiother Oncol 2018;126:339-46.

6. Lee SR, Kim MJ, Park SH, et al. Radiobiological and physical effects of patient setup errors during whole breast irradiation. Int J Radiat Res 2017;15:343-52.

7. Zhang X, Zhao KL, Guerrero TM, et al. FourDimensional computed tomography-based treatment planning for intensity modulated radiation therapy and proton therapy for distal esophageal cancer. Int J Radiat Oncol Biol Phys 2008;72:278-87.

8. Naseer A, Sankar V, Kate J, et al. Dose Motion Assessment with 4-Dimensional computed tomographic imaging for Non-Small cell lung cancer radiotherapy improve target volume coverage? Clin Med Insights Oncol 2017;11:1-7.

9. Duma MN, Berndt J, Rondak IC, et al. Implications of free breathing motion assessed by $4 \mathrm{D}$-computed tomography on the delivered dose in radiotherapy for esophageal 
cancer. Med Dosim 2015;40:378-82.

10. Khadige M, Salleron J, Marchesi V, et al. Cyberknife® stereotactic radiation therapy for stage I lung cancer and pulmonary metastases: evaluation of local control at 24 months. J Thorac Dis 2018;10:4976-84.

11. Iyengar P, Timmerman D. Stereptactic ablative radiotherapy for non-small cell lung cancer: rational and outcomes. J Natl Compr Canc Netw 2012;10:1514-20.

12. Shirato H, Seppenwoolde Y, Kitamura K, et al. Intrafractional tumor motion: lung and liver. Semin Radiat Oncol 2004;14:10-8.

13. Giraud P, Yorke E, Jiang S, et al. Reduction of organ motion effects in IMRT and conformal 3D radiation delivery by using gating and tracking techniques. Cancer Radiother 2006;10:269-82.

14. Mageras GS, Pevsner A, Yorke ED, et al. Measurement of lung tumor motion using respiration-correlated CT. Int J Radiat Oncol Biol Phys 2004;60:933-41.

15. Underberg RW, Lagerwaard FJ, Slotman BJ, et al. Use of maximum intensity projections (MIP) for target volume generation in 4DCT scans for lung cancer. Int J Radiat Oncol Biol Phys 2005;63:253-60.

16. Starkschall G, Forster KM, Kitamura K, et al. Correlation of gross tumor volume excursion with potential benefits of respiratory gating. Int J Radiat Oncol Biol Phys 2004;60:1291-7.

17. Shih HA, Jiang SB, Aljarrah KM, et al. Internal target volume determined with expansion margins beyond composite gross tumor volume in three-dimensional conformal radiotherapy for lung cancer. Int J Radiat Oncol Biol Phys 2004;60:613-22.

18. Underberg RW, Lagerwaard FJ, Cuijpers JP, et al. Four-dimensional CT scans for treatment planning in

Cite this article as: Wang G, Zhu X, Zhang F, Liu J, Yue L, Deng L, Zhou M. Applied research of a four-dimensional CT localization technique in radiotherapy and treatment planning for early lung cancer. Transl Cancer Res 2020;9(11):7227-7235. doi: $10.21037 /$ tcr-20-2800 stereotactic radiotherapy for stage I lung cancer. Int J Radiat Oncol Biol Phys 2004;60:1283-90.

19. Stroom JC, Heijmen BJ. Geometrical uncertainties, radiotherapy planning margins, and the ICRU-62 report. Radiother Oncol 2002;64:75-83.

20. Muirhead R, Mcnee SG, Featherstone C, et al. Use of maximum intensity projection (Mips) for target outing in 4DCT radiotherapy planning. J Thorac Oncol 2008;3:1433-8.

21. Huang L, Park K, Boike T, et al. A study on the dosimetric accuracy of treatment planning for stereotactic body radiation therapy of lung cancer using average and maximum intensity projection images. Radiother Oncol 2010;96:48-54.

22. Franks KN, Purdie TG, Dawson LA, et al. Incorporating heterogeneity correction and 4D CT in lung stereotactic body radiation therapy (SBRT): the effect on target coverage, organ-at-risk doses, and dose conformity. Med Dosim 2010;35:101-7.

23. Caldwell CB, Mah K, Skinner M, et al. Can PET provide the $3 \mathrm{D}$ extent of tumor motion for individualized internal target volumes? A phantom study of the limitations of CT and the promise of PET. Int J Radiat Oncol Biol Phys 2003;55:1381-93.

24. Vedam SS, Keall P, Kini VR, et al. Acquiring a fourdimensional computed tomography dataset using an external respiratory signal. Phys Med Biol 2003;48:45-62.

25. Keall P. 4-dimensional computed tomography imaging and treatment planning. Semin Radiat Oncol 2004;14:81-90.

26. Keall PJ, Starkschall G, Shukla H, et al. Acquiring 4D thoracic CT scans using a multislice helical method. Phys Med Biol 2004;49:2053-67. 